\title{
Conduta na lesão dural intraoperatória em artrodese da coluna lombar
}

\author{
Management of intraoperatory dural tear in lumbar spine arthrodesis \\ Conducta en lesión de duramadre intraoperatoria en artrodesis de la \\ columna lumbar
}

\author{
Fabiano de Mendonça Grandese' \\ Clóvis Yamazato² \\ Fábio Mastromauro de Oliveira ${ }^{2}$ \\ Iberê Ribeiro² \\ José Olympio Catão Bastos Júnior ${ }^{2}$ \\ René Kusabara ${ }^{3}$
}

\section{RESUMO}

Objetivo: estudo retrospectivo de pacientes com lesão dural intraoperatória de cirurgia da coluna vertebral lombar, conduzido por meio de um protocolo independente da extensão da lesão, complicações associadas e resultados após seguimento mínimo de um ano. Métodos: um total de 10 pacientes com durotomia acidental em cirurgias de descompressão e artrodese da coluna vertebral lombar, no período de Janeiro de 2007 a Janeiro de 2009, para tratamento de doença degenerativa, tiveram seus prontuários revisados, após terem sido tratados por meio de um protocolo embasado em reparo primário da lesão, drenagem subaracnoidea fechada, subfascial e subcutânea aspirativa realizados por cirurgião experiente, associado a repouso relativo com mobilidade precoce e terapia medicamentosa. Suas anotações e exames radiológicos foram verificados com seguimento pós-operatório e direcio-

\section{ABSTRACT}

Objective: a retrospective study of patients with spinal dural intraoperative lumbar spine surgery, conducted by means of a protocol regardless of the extent of the injury, associated complications and followup results after a minimum of one year. Methods: a total of 10 patients with accidental durotomy in surgical decompression and arthrodesis of the lumbar spine during the period from January 2007 to January 2009 for treatment of degenerative disease had their records reviewed after being treated by a protocol based in primary repair of the lesion, closed subarachnoid drainage, subfascial and subcutaneous aspiration drainage performed by experienced surgeons, combined with relative rest with early mobilization and drug therapy. Their notes and imaging were verified with follow-up aimed to identify symptoms that could suggest complications. Results: all
\end{abstract}

\section{RESUMEN}

Objetivo: un estudio retrospectivo de pacientes con lesión de la duramadre intraoperatoria de cirugía en la columna lumbar, llevada a cabo por medio de un protocolo independiente de la extensión de la lesión, complicaciones asociadas y los resultados de seguimiento después de un mínimo de un año. Métodos: 10 pacientes con durotomía accidental en la descompresión quirúrgica y artrodesis de la columna vertebral lumbar, en el período de enero de 2007 a enero de 2009, para el tratamiento de enfermedades degenerativas, tuvieron sus registros revisados después de tendieren sido tratados por un protocolo basado en la reparación primaria de la lesión, subaracnoidea drenaje cerrado, aspiración subfascial y subcutánea realizada por cirujanos con experiencia, combinada con el reposo relativo, con la movilización precoz y el tratamiento farmacológico. Sus notas y las imágenes fueron verificadas

\footnotetext{
Trabalho realizado no Grupo de Coluna do Hospital Municipal Dr. Cármino Caricchio - São Paulo (SP), Brasil; Hospital IFOR Ortopedia e Traumatologia - São Bernardo do Campo (SP), Brasil; Hospital IGESP - São Paulo (SP), Brasil.

'Estagiário de Coluna do Hospital Municipal Dr. Cármino Caricchio - São Paulo (SP), Brasil; Hospital IFOR Ortopedia e Traumatologia - São Bernardo do Campo (SP), Brasil; Hospital IGESP - São Paulo (SP), Brasil.

${ }^{2}$ Assistente de Coluna do Hospital Municipal Dr. Cármino Caricchio - São Paulo (SP), Brasil; Hospital IFOR Ortopedia e Traumatologia - São Bernardo do Campo (SP), Brasil; Hospital IGESP - São Paulo (SP), Brasil.

${ }^{3}$ Chefe do Grupo de Coluna do Hospital Municipal Dr. Cármino Caricchio - São Paulo (SP), Brasil; Hospital IFOR Ortopedia e Traumatologia - São Bernardo do Campo (SP), Brasil; Hospital IGESP - São Paulo (SP), Brasil.
} 
nados para identificação de sintomas sugestivos de complicações. Resultados: todos os pacientes apresentaram boa evolução, sem ocorrência de fístula liquórica ou infecção pós-operatória; três deles apresentaram cefaleia pós-operatória de leve intensidade. Não houve necessidade de reoperação em nenhum desses pacientes. Conclusão: concluiu-se que a conduta adotada é segura e de bom resultado para os pacientes quando ocorre esse tipo de lesão, independentemente da extensão da mesma. patients had a good outcome, without occurrence of cerebrospinal fluid leaks or postoperative infection; three had postoperative headache of mild intensity. There was no need for reoperation in these patients. Conclusion: we concluded that the conduct adopted is safe and has a good outcome for patients when this type of injury occurs, regardless of its extent.

\author{
KEYWORDS: Dura Mater/ \\ surgery; Subdural effusion/ \\ therapy; Spinal fusion/ \\ instrumentation; Reoperation; \\ Headache/cerebrospinal fluid
}

con el seguimiento postoperatorio y direccionadas para la identificación de síntomas sugestivos de complicaciones. Resultados: todos los pacientes tuvieron buena evolución, sin registro de pérdidas de líquido cefalorraquideo o infección postoperatoria, tres tuvieron cefalea en el postoperatorio de intensidad leve. No hubo necesidad de reintervención en esos pacientes. Conclusión: la conducta adoptada es segura y con buen resultado para pacientes en los que este tipo de lesión se produce, independiente de la extensión de la misma.

\section{DESCRIPTORES: Duramadre/} cirugía; Efusión subdural/ terapia; Fusión vertebral/ instrumentación; Reoperación; Cefalea/líquido cefalorraquídeo

\section{INTRODUÇÃO}

Ao mesmo tempo em que ocorre um incremento no número de procedimentos cirúrgicos na coluna, aumentase o grau de complexidade, favorecendo o aumento da incidência e da prevalência de lesões durais ${ }^{1}$, segunda complicação mais frequente associada a sequelas de cirurgia da coluna com implicações médico-legais, não podendo ser considerado um evento totalmente benigno ${ }^{2}$.

A lesão intraoperatória da dura-máter é causa de vários transtornos intra e pós-operatórios, como aumento do tempo de cirurgia, formação de fístula liquórica e pseudomeningocele, podendo ocasionar também déficit neurológico, infecções do sítio cirúrgico e do sistema nervoso central (SNC) etc. ${ }^{1-21}$.

Existem dados que sugerem que os cirurgiões de coluna tendem a subestimar a frequência desse tipo de complicação ${ }^{3}$, pois, com exceção de casos de fístula liquórica com saída de líquido cerebrospinhal pela pele, infecções do SNC ou presença de fotofobia, muitos casos de pseudomeningocele após durotomia incidental não reconhecida no intraoperatório são assintomáticos ou podem ser mascarados na presença de cefaleia postural e outras algias controladas por medicação analgésica, frequentemente utilizada pelos pacientes tanto no pré quanto no pós-operatório. Estudos revelam variação da incidência de 0 a 17,4\% em amostras que vão de 5 a 338 casos de acordo com o diagnóstico primário, o tipo e a complexidade do procedimento cirúrgico ou o número de cirurgias prévias na coluna desses pacientes $^{3,4,6,7,9,12,13,17,18}$, entre outros fatores, como idade avançada ou outras comorbidades, bem como o nível de treinamento do cirurgião ${ }^{5}$ ou o uso de perfurador de alta rotação ${ }^{6}$.

Nas três maiores séries descritas na literatura, Khan et al., em uma série de 3.183 pacientes com 338 lesões durais, encontraram uma incidência de 7,6\% em cirurgias primárias e $15,9 \%$ em cirurgias de revisão ${ }^{7}$. Cammisa et al., em uma série de 2.144 pacientes com 74 lesões durais, encontraram incidência de 3,1\% em cirurgias de descompressão simples, de 1\% em procedimentos de descompressão e fusão sem instrumentação, de $2 \%$ na descompressão com instrumentação e de $8,1 \%$ em cirurgias de revisão da coluna vertebral ${ }^{8}$. Tafazal et al., numa série de 93 pacientes com lesões durais, evidenciaram que a incidência era de 3,5\% em discectomia primária, 8,5\% em estenose do canal e 13,2\% em cirurgias de revisão ${ }^{3}$.

De acordo com a base de dados encontrada na literatura, o tratamento da lesão dural consiste no emprego de diversas técnicas, tais como: repouso em decúbito Trendelemburg; reparo da lesão com injeção intratecal de matriz de colágeno sintética percutânea ou de bloodpatch; selante de fibrina, hemostático gelfoam ou surgicel; sutura associada a enxerto de gordura, de músculo e de fáscia, podendo ser associada a repouso em decúbito no leito por tempo prolongado ou mobilização precoce; instalação ou não de drenagem gravitacional subaracnoidea, gravitacional ou aspirativa subfascial e/ou subcutânea; além do uso de medicações e reoperações, 
de forma isolada ou aglutinadas por meio de protocolos não consensuais, muitas vezes conflitantes entre si, com resultados parcialmente satisfatórios ${ }^{1,3,5-16,21}$.

O objetivo de nosso estudo foi analisar os resultados do tratamento de 10 pacientes com lesões durais ocorridas acidentalmente em cirurgias da coluna vertebral lombar, com base na adoção de um protocolo elaborado por meio de dados encontrados na literatura e modificado pela experiência profissional de nossa equipe. Sugerimos um método de tratamento para esse tipo de lesão, independentemente de sua extensão.

\section{MÉTODOS}

Este estudo trata de uma análise retrospectiva de 10 pacientes operados pela nossa equipe em um período de dois anos, compreendido entre Janeiro de 2007 e Janeiro de 2009, com seguimento pós-operatório mínimo de um ano, que apresentaram lesão dural intraoperatória em cirurgias da coluna lombar com doença degenerativa discal, com média de idade de 47 anos, sendo quatro pacientes do sexo masculino e seis pacientes do sexo feminino (Tabela 1).

Todos os pacientes foram submetidos à artrodese com o uso de parafusos pediculares, e os níveis operados foram quatro L4-S1, dois L3-S1, dois L4-L5, um L5-S1 e um L3-L4. Três pacientes foram submetidos à cirurgia primária e sete já haviam sido submetidos à cirurgia prévia.

Uma das lesões da dura-máter foi causada por escorregamento do parafuso pedicular durante seu posicionamento inicial. Os outros nove pacientes sofreram a lesão durante a fase de descompressão do canal vertebral.

Os pacientes foram submetidos a reparo da lesão mediante sutura interrompida por meio de pontos simples com fio de seda 5.0 associado à enxertia de gordura, músculo ou fáscia, dependendo da extensão da lesão, ou após extravasamento de líquor no teste com manobras de Valsalva intraoperatória, com utilização de cola de fibrina (Beriplast R) em cinco pacientes após a sutura. Nos outros casos, não houve disponibilidade desse material no hospital.

Inserimos um dreno aspirativo multifenestrado subfascial e outro subcutâneo, com espessura de 3,2 $\mathrm{mm}$, ambos conectados ao mesmo coletor aspirativo em sanfona. Como convenção, adotamos que o dreno subfascial era exposto em posição proximal ao superficial na pele, a fim de facilitar a ordem de retirada dos mesmos. Após a finalização da cirurgia, foi introduzido um cateter subaracnoideo através de agulha peridural em nível proximal à via de acesso e conectado a uma bolsa de transferência estéril, por meio de equipo de soro sob sistema fechado, posicionada no mesmo nível do paciente em seu leito.

Os pacientes permaneceram com sondagem vesical de demora para evitar a necessidade de aumento da mobilização no leito. Foi administrada acetazolamida (Diamox) e antibioticoprofilaxia com cefazolina.

Os pacientes foram mantidos em repouso absoluto por três dias, com elevação do decúbito apenas para alimentação, além de decúbito em posição de Trendelenburg noturno. No terceiro dia de pós-operatório, os pacientes foram encaminhados para banho no chuveiro, mantendo-se ainda o repouso relativo, com retirada da sondagem vesical. O cateter subaracnoideo era fechado durante o tempo em ortostatismo para diminuir a drenagem liquórica.

No quarto dia após a cirurgia, foi retirado o cateter subaracnoideo, observando-se o volume residual do dreno aspirativo.

No quinto dia, foi retirado o dreno aspirativo subfascial, e o dreno superficial no dia seguinte; foi dada alta hospitalar caso não houvesse maiores sintomas. A média de internação foi de 6,8 dias.

Todos os pacientes foram orientados a manter o uso de acetazolamida por mais 15 dias, além da elevação dos pés da cama pelo mesmo período em sua residência (Trendelemburg).

TABELA 1 - Pacientes com lesão dural intraoperatória de cirurgia da coluna lombar e a presença de sintomas sugestivos de persistência da durotomia no seguimento de um ano

\begin{tabular}{|c|c|c|c|c|c|c|c|c|c|c|c|c|}
\hline Paciente & Idade & Sexo & Cirurgia & $\begin{array}{c}\text { Nível de } \\
\text { artrodese }\end{array}$ & $\begin{array}{l}\text { Cola de } \\
\text { fibrina }\end{array}$ & $\begin{array}{c}\mathrm{PO} \\
\mathrm{I}\end{array}$ & $\begin{array}{l}\mathrm{PO} \\
15 \\
\end{array}$ & $\begin{array}{l}\mathrm{PO} \\
45 \\
\end{array}$ & $\begin{array}{l}\mathrm{PO} \\
3 \mathrm{~m}\end{array}$ & $\begin{array}{l}\mathrm{PO} \\
6 \mathrm{~m}\end{array}$ & $\begin{array}{l}\mathrm{PO} \\
1 \mathrm{a}\end{array}$ & $\begin{array}{l}\text { ALTA } \\
\text { (PO) }\end{array}$ \\
\hline ARM & 34 & $M$ & Primária & L4-L5 & $S$ & - & - & - & - & - & - & 6 \\
\hline BSA & 42 & M & Revisão & L4-S 1 & S & C & $C$ & - & - & - & - & 6 \\
\hline BTMN & 23 & F & Primária & L4-S 1 & $N$ & - & - & - & - & - & - & 6 \\
\hline CAFO & 34 & $M$ & Revisão & L3-S I & S & - & - & - & - & - & - & 6 \\
\hline CEFR & 67 & F & Revisão & L5-S 1 & S & $C$ & - & - & - & - & - & 8 \\
\hline EMO & 29 & $F$ & Revisão & L4-S 1 & $N$ & - & & - & - & - & - & 6 \\
\hline EMXA & 44 & F & Revisão & L4-S 1 & $N$ & & - & - & - & - & & 6 \\
\hline$H Y$ & 72 & M & Revisão & L3-S I & S & & - & - & - & - & & 10 \\
\hline $\mathrm{MJOL}$ & 57 & F & Primária & L4-L5 & S & C & $C$ & - & - & - & - & 6 \\
\hline MAP & 69 & $\mathrm{~F}$ & Revisão & L3-L4 & S & - & - & - & - & - & - & 8 \\
\hline
\end{tabular}

M: masculino; F: feminino; S: sim; N: não; C: cefaleia; -: assintomático. 


\section{RESULTADOS}

Os pacientes foram orientados a retornar em duas semanas para retirada dos pontos da cirurgia, quando foram orientados a deixar a posição de Trendelemburg domiciliar. A analgesia foi mantida nesse período por meio do uso de anti-inflamatórios e analgésicos que continham codeína, com diminuição gradativa, conforme os sintomas do paciente.

Não houve sinal de fístula liquórica em nenhum dos pacientes. Três deles desenvolveram sintoma de cefaleia postural leve durante a internação, que persistiu em um dos pacientes por mais uma semana.

Os retornos pós-operatórios foram realizados com seis semanas, três meses, seis meses e um ano de pós-operatório, sempre com solicitação de novas radiografias para controle e evolução da consolidação, que ocorreu em todos os pacientes.

Nenhum dos pacientes desenvolveu infecção superficial ou profunda ou sequela neurológica. Dois apresentaram parestesia temporária, regredindo após seis semanas, sem ter sido necessária nenhuma outra intervenção cirúrgica.

\section{DISCUSSÃO}

Estudos publicados recentemente defendem que a lesão dural reconhecida no ato cirúrgico deve ser reparada no mesmo procedimento ${ }^{6,10-12}$, com resultados semelhantes quando comparados a pacientes operados sem lesões durais em longo prazo $3,7,9,13$, enquanto Eismont et al. demonstraram mal resultado no tratamento não cirúrgico ${ }^{11}$. Cammisa et al. também relataram que o único paciente com lesão dural não identificada no intraoperatório teve sua evolução pós-cirúrgica complicada por crises de cefaleia postural persistente ${ }^{8}$; Entretanto, em estudo prospectivo com seguimento de 10 anos, Saxler et al. relataram que pacientes que tinham sofrido lesão dural apresentaram aumento das taxas de cefaleia e lombalgia em relação a indivíduos controle; porém, não foi utilizada drenagem subfascial ou subaracnoidea em seu estudo ${ }^{14}$.

Narotam et al. recentemente relataram $95 \%$ de sucesso com reparo sem sutura usando uma técnica de enxerto de matriz de colágeno sintético em uma série de 110 pacientes $^{15}$, enquanto Patel et al. relataram apenas $50 \%$ de sucesso no reparo de lesões durais com técnica de injeção percutânea de cola de fibrina guiada por tomografia computadorizada em seis pacientes ${ }^{16}$.

O uso de drenos foi desaconselhada no passado por Eismont et al. Com base em seu estudo de apenas cinco lesões da dura-máter, favoreceriam a formação de fístula mielocutânea ${ }^{11}$, cuja posição também foi defendida por Bosacco et al ${ }^{1}$.

Em um estudo realizado por Kitchel et al. com 19 lesões durais, foi realizada drenagem subaracnoidea lombar fechada; porém, $58 \%$ dos pacientes desenvolveram sintomas como náuseas e vômitos, e, em quatro pacientes, foi necessária nova intervenção ${ }^{12}$.

Khan et al. utilizaram um protocolo baseado em reparo do defeito com sutura contínua ancorada e drenagem subfascial aspirativa associado à mobilização precoce. Os pacientes com persistência de sintomas após o sexto dia foram conduzidos à reoperação para desbridamento, reparo da lesão com sutura e uso de selante de tecido caso persistisse o extravasamento. Houve colocação de drenagem subfascial associada à drenagem subaracnoidea somente nos casos de persistência de vazamento de líquor após a segunda exploração, com $98,2 \%$ de sucesso, e $1,8 \%$ dos pacientes necessitaram de reoperação, evidenciando a utilidade da drenagem subfascial para impedir o acúmulo de líquor, que favorece o desenvolvimento de fístula ${ }^{7}$.

Wang et al. descreveram que o uso de drenagem subaracnoidea pode ser apropriado em pacientes com lesão dural, embora não a tenham utilizado em seu estudo?.

Com base nos estudos de Cain et al. sobre a fisiologia do reparo da lesão dural em modelos caninos, a formação de pontes de fibrina ocorre até o sexto dia após o reparo da lesão, motivo pelo qual mantivemos a drenagem profunda em nossos pacientes. A cicatrização ocorreu por volta do décimo $\mathrm{dia}^{22}$ e mantivemos a acetazolamida por mais duas semanas. A inibição da anidrase carbônica no plexo coroide reduziu a produção de líquor, diminuindo a pressão intracraniana.

Carlson et al. verificaram que a posição de decúbito supino reduz cerca de $46 \%$ da pressão intradural, reduzindo também a tensão na região do reparo da lesão ${ }^{21}$.

Em seu estudo, Cammisa et al. tiveram uma taxa de reoperações de $9,1 \%$ (6 de 66 pacientes $)^{8}$. Da mesma forma, Wang et al., em 2,3\% (2 de 88 pacientes $)^{9}$, e Khan et al. em 1,8\% (6 dos 338 pacientes), tendo qualificado seu protocolo como altamente eficaz ${ }^{7}$. Em nosso estudo, nenhum paciente foi submetido a novo procedimento cirúrgico, reduzindo a morbidade pós-operatória.

\section{CONCLUSÃO}

Devemos sempre atentar aos fatores evidenciados, que produzem aumento de risco na incidência de lesão dural iatrogênica, a fim de redobrar a atenção intraoperatória e minimizar a morbidade pós-operatória.

Embora nossa casuística não tenha significância estatística $(n=10)$ e apesar de o procedimento ser mais invasivo, devido ao uso de cateter subaracnoideo, nosso protocolo de manejo intra e pós-operatório do paciente com lesão dural, independentemente do tamanho da lesão, não apresentou aumento do risco infeccioso ou neurológico e foi eficaz em $100 \%$ dos casos, sem a necessidade de reexploração da lesão, demonstrando-se promissor no tratamento de lesões durais intraoperatórias. 


\section{REFERÊNCIAS}

1. Bosacco SJ, Gardner MJ, Guille JT. Evaluation and treatment of dural tears in lumbar spine surgery: a review. Clin Orthop Relat Res. 2001;(389):238-47.

2. Goodkin R, Laska LL. Unintended "incidental" durotomy during surgery of the lumbar spine: medicolegal implications. Surg Neurol. 1995;43(1):4-12.

3. Tafazal SI, Sell PJ. Incidental durotomy in lumbar spine surgery: incidence and management. Eur Spine J. 2005;14(3):287-290.

4. McCormack BM, Zide BM, Kalfas, IH. Cerebrospinal fluid fistula and pseudomeningocele after spine surgery. In: Benzel EC, editor. Spine surgery: Techniques, complication avoidance and management. Philadelphia: Churchill Livingstone; 1999. p. 1465-74.

5. Sin AH, Caldito G, Smith D, Rashidi M, Willis B, Nanda A. Predictive factors for dural tear and cerebrospinal fluid leakage in patients undergoing lumbar surgery. J Neurosurg Spine. 2006;5(3):224-7.

6. Hodges SD, Humphreys CS, Eck JC, Covington LA. Management of incidental durotomy without mandatory bed rest: a retrospective review of 20 cases. Spine (Phila $\mathrm{Pa}$ 1976). 1999;24(19):2062-4.

7. Khan MH, Rihn J, Steele G, Davis R, Donaldson WF 3rd, Kang JD, et al. Postoperative management protocol for incidental dural tears during degenerative lumbar spine surgery: a review of 3,183 consecutive degenerative lumbar cases. Spine (Phila Pa 1976). 2006;31(22):2609-13.

8. Cammisa FP Jr, Girardi FP, Sangani PK, Parvataneni HK, Cadag S, Sandhu HS. Incidental durotomy in spine surgery. Spine (Phila Pa 1976). 2000;25(20):2663-7.
9. Wang JC, Bohlman HH, Riew DK. Dural tears secondary to operations on the lumbar spine: Management and results after a two-yearminimum follow-up of eighty-eight patients. J Bone Joint Surg Am. 1998;80(12):1728-32.

10.Finnegan WJ, Fenlin JM, Marvel JP, Nardini RJ, Rothman RH. Results of surgical intervention in the symptomatic multiply-operated back patient. Analysis of sixty-seven cases followed for three to seven years. J Bone Joint Surg Am. 1979;61(7):107782.

11.Eismont FJ, Wiesel SW, Rothman RH. Treatment of dural tears associated with spinal surgery. J Bone Joint Surg Am. 1981;63(7):1132-6.

12.Kitchel SH, Eismont FJ, Green BA. Closed subarachnoid drainage for management of cerebrospinal fluid leakage after an operation on the spine. J Bone Joint Surg Am. 1989;71(7):984-7.

13.Jones AA, Stambough JL, Balderston RA, Rothman RH, Booth RE Jr. Longterm results of lumbar spine surgery complicated by unintended incidental durotomy. Spine (Phila Pa 1976). 1989;14(4):443-6.

14.Saxler G, Krämer J, Barden B, Kurt A, Pförtner J, Bernsmann K. The long-term clinical sequelae of incidental durotomy in lumbar disc surgery. Spine (Phila Pa 1976). 2005;30(20):2298-302.

15.Narotam PK, José S, Nathoo N, Taylon C, Vora Y. Collagen matrix (DuraGen) in dural repair: analysis of a new modified technique. Spine (Phila Pa 1976). 2004;29(24):2861-7.
16.Patel MR, Louie W, Rachlin J. Postoperative cerebrospinal fluid leaks of the lumbosacral spine: management with percutaneous fibrin glue. AJNR Am J Neuroradiol. 1996;17(3):495500.

17. Stolke D, Sollmann WP, Seifert V. Intra- and postoperative complications in lumbar disc surgery. Spine (Phila $\mathrm{Pa}$ 1976). 1989;14(1):56-9.

18. Wiesel SW. The multiply operated lumbar spine. Instr Course Lect. 1985;34:68-77.

19.Miller PR, Elder FW Jr. Meningeal pseudocysts (meningocele spurius) following laminectomy. Report of ten cases. J Bone Joint Surg Am. 1968;50(2): 268-76.

20.Nash CL Jr, Kaufman B, Frankel VH. Postsurgical meningeal pseudocysts of the lumbar spine. Clin Orthop Relat Res. 1971;(75):167-78.

21.Carlson GD, Oliff HS, Gorden C, Smith J, Anderson PA. Cerebral spinal fluid pressure: effects of body position and lumbar subarachnoid drainage in a canine model. Spine (Phila Pa 1976). 2003;28(2):119-22.

22.Cain JE Jr, Lauerman WC, Rosenthal HG, Broom MJ, Jacobs RR. The histomorphologic sequence of dural repair. Observations in the canine model. Spine (Phila Pa 1976). 1991;16(8 Suppl):S319-23
Correspondência
Fabiano de Mendonça Grandese
Rua Corta Vento, 28
CEP $03313-010$
Tel: 11 1) 3542-3403
E-mail: famegran@ig.com.br 